Article

\title{
First-Principles Study on the Stabilities, Electronic and Optical Properties of $\mathrm{Ge}_{\mathrm{x}} \mathrm{Sn}_{1-\mathrm{x}}$ Se Alloys
}

\author{
Qi Qian, Lei Peng, Yu Cui, Liping Sun, Jinyan Du and Yucheng Huang *
}

College of Chemistry and Material Science, The Key Laboratory of Functional Molecular Solids, Ministry of Education, Anhui Laboratory of Molecule-Based Materials, Anhui Normal University, Wuhu 241000, China; qianq@ahnu.edu.cn (Q.Q.); penglei@ahnu.edu.cn (L.P.); cuiyu@ahnu.edu.cn (Y.C.); sunlp@ahnu.edu.cn (L.S.); dujinyn@mail.ahnu.edu.cn (J.D.)

* Correspondence: huangyc@mail.ahnu.edu.cn; Tel.: +86-553-3869303

Received: 25 September 2018; Accepted: 17 October 2018; Published: 25 October 2018

\begin{abstract}
We systematically study, by using first-principles calculations, stabilities, electronic properties, and optical properties of $\mathrm{Ge}_{x} \mathrm{Sn}_{1-\mathrm{x}} \mathrm{Se}$ alloy made of SnSe and GeSe monolayers with different Ge concentrations $x=0.0,0.25,0.5,0.75$, and 1.0. Our results show that the critical solubility temperature of the alloy is around $580 \mathrm{~K}$. With the increase of Ge concentration, band gap of the alloy increases nonlinearly and ranges from 0.92 to $1.13 \mathrm{eV}$ at the PBE level and 1.39 to $1.59 \mathrm{eV}$ at the HSE06 level. When the Ge concentration $\mathrm{x}$ is more than 0.5 , the alloy changes into a direct bandgap semiconductor; the band gap ranges from 1.06 to $1.13 \mathrm{eV}$ at the PBE level and 1.50 to $1.59 \mathrm{eV}$ at the HSE06 level, which falls within the range of the optimum band gap for solar cells. Further optical calculations verify that, through alloying, the optical properties can be improved by subtle controlling the compositions. Since $\mathrm{Ge}_{x} \mathrm{Sn}_{1-x} \mathrm{Se}$ alloys with different compositions have been successfully fabricated in experiments, we hope these insights will contribute to the future application in optoelectronics.
\end{abstract}

Keywords: GeSe monolayer; SnSe monolayer; alloy; first-principles; electronic property; optical property

\section{Introduction}

Since the emergence of grapheme [1], two-dimensional (2D) materials have attracted intense attention in the scientific community due to richness of the physical properties [2]. It is known that although graphene has high conductivities, the feature of having a zero band gap greatly restricts its application in the semiconductor industry [3]. As important supplements of graphene [4,5], 2D layered materials such as transition-metal dichalcogenides (TMDs) [6], black phosphorene (BP) [7], hexagonal boron nitride (h-BN) [8], metal carbides and carbonitrides (MXenes) [9], and monoelemental arsenene, antimonene [10], bismuthene [11], silicone [12], germanene [13], tellurene [14], etc., have been experimentally manufactured or theoretically predicted which exhibit unique electronic and optical properties for broad applications at the nanoscales. Versatile and complementary properties of these 2D materials can meet a large variety of requirements for potential application. A typical example is $\mathrm{MoS}_{2}$ [15-17] that has attracted tremendous attention among the TMDs materials. While it has a large intrinsic band gap of $1.8 \mathrm{eV}$ [18], the reported mobilities are only in the range of $0.5-3 \mathrm{~cm}^{2} \mathrm{~V}^{-1} \mathrm{~s}^{-1}$, which are too low for practical application in electronic devices [4]. Recently, the booming 2D materials of $\mathrm{BP}$ seems to make up the gap between graphene and $\mathrm{MoS}_{2}$ because it has a tunable direct band gap from $0.3 \mathrm{eV}$ of the bulk to $1.5 \mathrm{eV}$ of the monolayer [19]. The resulted high theoretical mobility [7,19], excellent near-infrared properties [20] as well as high photoelectric conversion capacity [21] endow its widely potential application in the field of electronic and optical devices. However, the stability 
of phosphorene in air and water needs to be enhanced. Thus, it seems that the single-component materials always have some disadvantages that greatly affect their widespread applications.

Alloyed 2D semiconductors can display compositionally tunable properties, which distinct from both their bulk alloys and binary alloy end-members. Through forming heterojunctions, the limitation of single-component 2D materials is expected to be broken through. Abundant researches on different kinds of the heterostructures, such as van der Wall (vdW) heterostrutures, lateral (in-plane) heterostructures and solid solution heterostrutures, have confirmed that the properties of single materials can be tailored by mixing with the second components, which provides a feasible way to improving the electronic, optoelectronic, as well as the catalytic properties of nanostructures [19,22-25]. For examples, the band gap of graphene is considerably open when vdW heterostructure of graphene/g- $\mathrm{C}_{3} \mathrm{~N}_{4}$ bilayer is formed [26]. Likewise, through forming $\mathrm{MoS}_{2}-\mathrm{WS}_{2}$ heterostructure, the carrier mobility is greatly enhanced to $65 \mathrm{~cm}^{2} \mathrm{~V}^{-1} \mathrm{~s}^{-1}$ [27]. Moreover, a facile and general method to passivate thin BP flakes with large-area high-quality monolayer h-BN sheets grown by the chemical vapor deposition (CVD) method was developed to preserve atomic layered BP flakes from degradation [28].

Recently, our group [29] has studied the electronic and optical properties of $\mathrm{SnSe}_{2 x} \mathrm{~S}_{2(1-x)}$ anion alloy through first-principles calculations. It was found that the band gap is not confined in a certain value, but varies in the range of the band gap of $\mathrm{SnSe}_{2}$ to that of $\mathrm{SnS}_{2}$, depending on the ratio of $\mathrm{SnSe}_{2}$ and $\mathrm{SnS}_{2}$. The adsorption strength is enhanced in the visible spectral region after alloying. Moreover, the alloys are predicted to be stable and would be favorably fabricated from the calculation results of phase diagram. Shortly after that, Wang et al. [30] have experimentally prepared $2 \mathrm{D} \mathrm{SnSe}_{2(1-\mathrm{x})} \mathrm{S}_{2 \mathrm{x}}$ alloys with five different $S$ compositions ( $x=0,0.25,0.5,0.75$, and 1$)$ by the chemical vapor transport (CVT) method. Different from the independent $\mathrm{SnSe}_{2}$ or $\mathrm{SnS}_{2}$ monolayer, carrier mobility of SnSeS field-effect transistor can be obviously increased by light illumination of $532 \mathrm{~nm}$ laser, indicative of the potential application as a phototransistor. Besides, different cation alloys, such as $\mathrm{Mo}_{1-\mathrm{x}} \mathrm{W}_{\mathrm{x}} \mathrm{S}_{2}, \mathrm{Mo}_{1-\mathrm{x}} \mathrm{W}_{\mathrm{x}} \mathrm{Se}_{2}$, etc., have also been successfully synthesized which exhibit different functions with respect to their end-members [31-33]. Therefore, novel properties can be obtained by alloying different 2D materials.

Due to high stability, earth abundance and environmental sustainability, 2D group IVA monochalcogenides (MXs), i.e., GeS, SnS, GeSe and SnSe [34-37], which are isostructural to black phase of phosphorene, have attracted intense attention recently. Experimentally, solid solutions can be formed with complete solid solubility among these MXs because of their structural similarity. Jannise et al [38]. have synthesized ternary $\mathrm{Sn}_{x} \mathrm{Ge}_{1-x}$ Se nanocrystals and exhibited adjustable components over the entire alloy range $(0 \leq x \leq 1)$. Compositional tuning on the lattice parameters, band gaps, and morphologies have been demonstrated and the alloy formation mechanism were thereby proposed. Moreover, Fu et al [39]. have successfully prepared Ge-doped SnSe polycrystals by the zone-melting combined with hot-pressing methods, and found that Ge is not an ideal dopant for optimizing the thermoelectric properties of SnSe. Since experimental investigations on the composite system made by GeSe and SnSe have been successfully carried out, however, as far as we know, theoretical investigations related to this system have not been reported yet.

In this contribution, stabilities, electronic structures and optical properties of single-layer $\mathrm{Ge}_{x} \mathrm{Sn}_{1-\mathrm{x}} \mathrm{Se}$ alloys with different Ge concentrations are systematically examined on the basis of density functional calculations. The soluble temperature of the alloy, variations of the electronic and optical properties as well as the underlying reasons are given. The potential application in optoelectronics is proposed.

\section{Computational Details}

First-principles calculations were carried out on the basis of density functional theory (DFT), as implemented in the Vienna Ab-initio Simulation Package (VASP) program [40,41]. The electron-ion interactions are descried by the projector-augmented plane wave (PAW) method [42]. Perdew-Burke-Ernzerhof (PBE) functional $[43,44]$ in generalized gradient approximation (GGA) was 
used to process the electron-exchange correlation interactions unless stated otherwise and a $450 \mathrm{eV}$ energy cutoff for the plane-wave basis sets were used. Vacuum height is set to $20 \AA$ along $x$-direction to avoid the interactions between two periodic repeating units. The convergence thresholds for energy and force were set to $10^{-5} \mathrm{eV}$ and $0.02 \mathrm{eV} / \AA$, respectively. The Brillouin zone was represented by a Monkhorst-Pack [45] special k-point mesh of $1 \times 3 \times 3$ for geometry optimizations, whereas a larger grid of $1 \times 5 \times 5$ was used for band structure computations.

\section{Results and Discussion}

\subsection{Structures and Stabilities of $\mathrm{Ge}_{x} \mathrm{Sn}_{1-x} \mathrm{Se}$ Alloys}

To best approximate the random solid solution, special quasi-random structures (SQSs) with five different Ge compositions ( $x=0,0.25,0.5,0.75$, and 1) of the $\mathrm{Ge}_{\mathrm{x}} \mathrm{Sn}_{1-\mathrm{x}} \mathrm{Se}$ alloys [46] were generated using the tool in the alloy theoretic automated toolkit (ATAT) [47]. Lattice parameters of each structure are then optimized, and a linear dependence on the composition is found, which is known as Vegard's law [48]. In Figure 1, three typical alloys with the Ge concentration of $0.25,0.50$ and 0.75 are illustrated. Note that phase stability of the alloy is critical to the possibility of alloy formation. To verify the structural stabilities of these alloys, a thermodynamic calculation was performed as previously proposed [29].

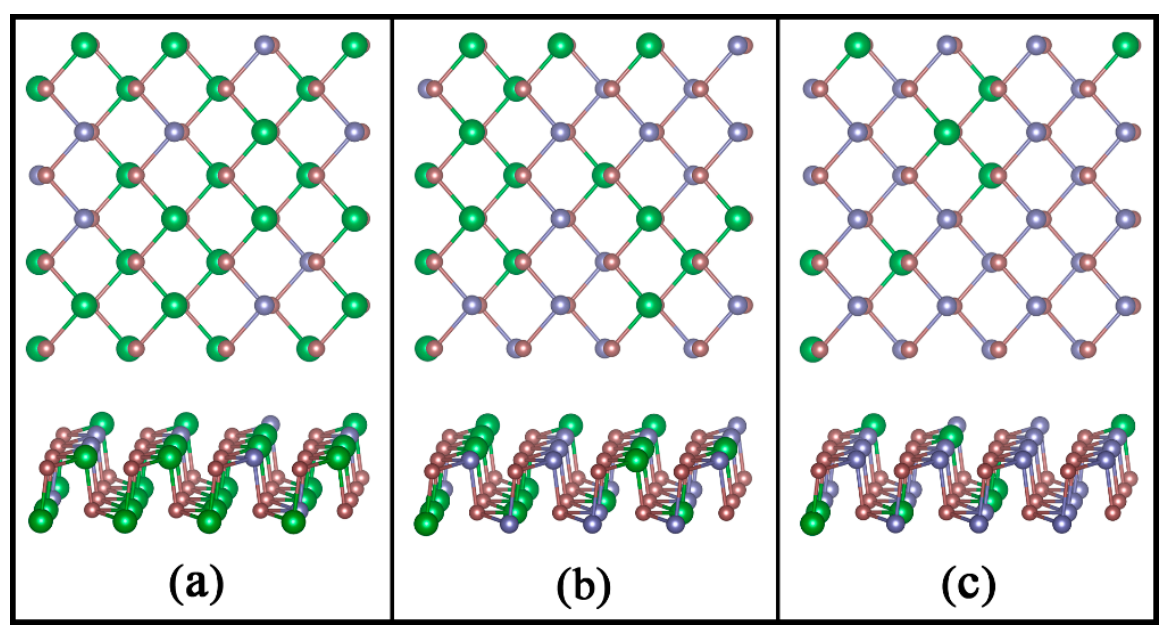

Figure 1. Top and Side Views of (a) $\mathrm{Ge}_{8} \mathrm{Sn}_{24} \mathrm{Se}_{32}$, (b) $\mathrm{Ge}_{16} \mathrm{Sn}_{16} \mathrm{Se}_{32}$ and (c) $\mathrm{Ge}_{24} \mathrm{Sn}_{8} \mathrm{Se}_{32}$ Monolayers with the Ge Concentration $x$ of $0.25,0.50$ and 0.75 , respectively.

First, the mixing enthalpy $\Delta H_{m}(x)$ is calculated through subtracting the energy summation of pure GeSe (A) and SnSe (B) from the total energy of $\mathrm{Ge}_{\mathrm{x}} \mathrm{Sn}_{1-\mathrm{x}} \mathrm{Se}$ monolayer. That is,

$$
\Delta H_{m}(x)=E\left(A_{x} B_{1-x}\right)-\left[x E_{A}+(1-x) E_{B}\right]
$$

In Figure 2a, the calculated mixing enthalpies as a function of Ge concentrations are shown. It is found that the binary alloys always have positive mixing enthalpies, showing a phase separation tendency at low temperatures. Considering the contribution of the mixed entropy, the solubility of the alloy would be enhanced by increasing the temperature. 

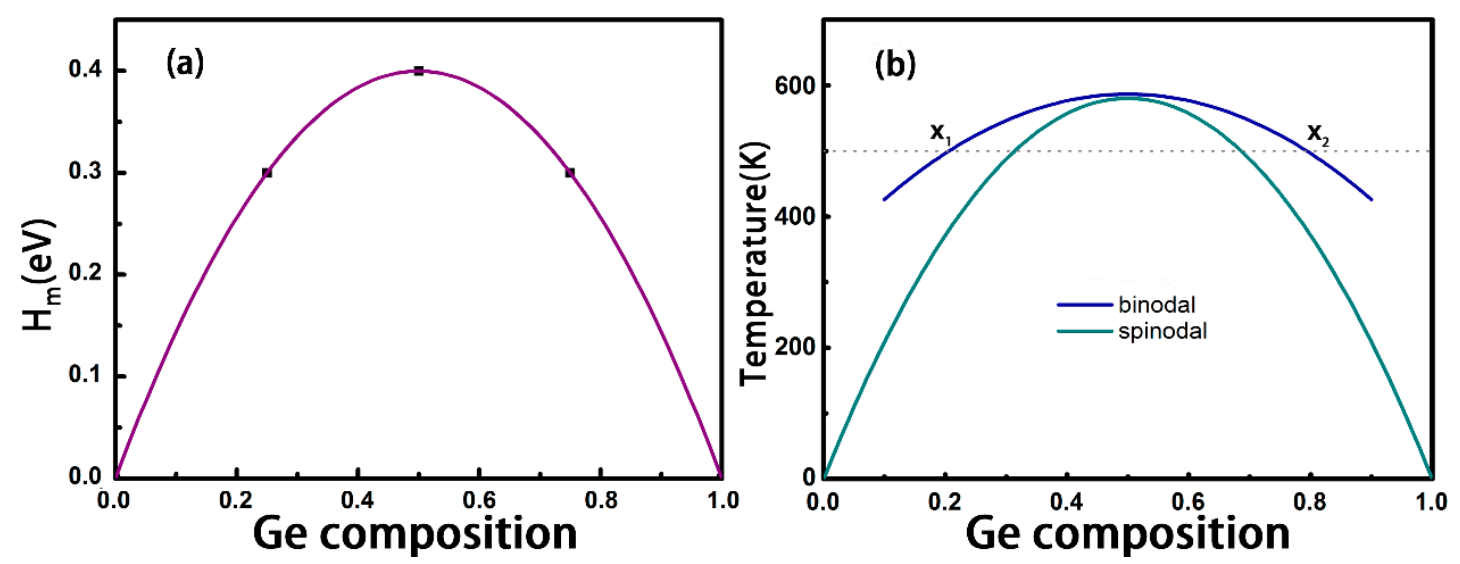

Figure 2. (a) Mixing Enthalpy and (b) Quasi-binary Phase Diagram as a Function of the Ge Content for the $\mathrm{Ge}_{\mathrm{x}} \mathrm{Sn}_{1-\mathrm{x}} \mathrm{Se}$ Alloys. Binodal and Spinodal Curves are Shown in Royal and Dark Cyan Lines in (b).

Here, the mixing entropy is calculated from a classical formula in the textbook [49]:

$$
\Delta S_{m}(x)=-2[x \ln x+(1-x) \ln (1-x)] k_{B},
$$

and the free energy of mixing is calculated corresponding to the following formula:

$$
\Delta F_{m}(x)=\Delta H_{m}(x)-T \Delta S_{m}(x) .
$$

To obtain the critical temperature of alloy mutual solubility, a second-order polynomial based on the quasi-chemical model is used to describe the relationship between mixing enthalpies and Ge concentrations:

$$
\Delta H_{m}(x)=\Omega x(1-x),
$$

where $\Omega$ is the interaction parameter dependent on the material. Accordingly, the mixing Helmholtz free energy can be rewritten as

$$
\Delta F_{m}(x)=\Omega x(1-x)+R T[x \ln x+(1-x) \ln (1-x)],
$$

in which the value of $\Delta F_{m}$ only relies on the Ge concentration $x$. Therefore, the binodal solubility curve and spinodal decomposition curve can be simply obtained by the formula of $\frac{\partial F_{m}}{\partial x}=0$ and $\frac{\partial^{2} F_{m}}{\partial x^{2}}=0$, respectively.

The obtained binary phase diagram is shown in Figure $2 b$, where we can see the binodal and spinodal curves meet at $\mathrm{x}=0.5$ at a critical temperature $T_{c}=\Omega / 2 R$. The symmetrical feature of the curve is consistent with the systems of $\mathrm{Mo}_{1-x} \mathrm{~T}_{x} \mathrm{~S}_{2}\left(\mathrm{~T}=\mathrm{W}, \mathrm{Cr}\right.$, and V) [17] and $\mathrm{SnSe}_{2(1-x)} \mathrm{S}_{2 x}$ [29]. The calculated critical temperature is $580 \mathrm{~K}$, which is an effortlessly realizable temperature in the laboratory, indicating that this kind of alloys can be favorably prepared. Note that if we count in the entropy contributed by lattice vibration, the solubility may be underestimated. In fact, the $\mathrm{Sn}_{\mathrm{x}} \mathrm{Ge}_{1-\mathrm{x}} \mathrm{Se}$ alloys have been successfully fabricated by Buckley et al., who heated precursor solutions to $500 \mathrm{~K}$ and held for $4.75 \mathrm{~h}$ with stirring [38].

As indicated in Figure 2b, when the temperature exceeds the critical temperature of $580 \mathrm{~K}$, the alloys become stable in the whole range of composition in thermodynamics. At variance, when the temperature is lower than $580 \mathrm{~K}$, for example, $500 \mathrm{~K}$, the alloys can be formed only when the concentration of Ge is in the ranges of $0<x<x 1$ and $x 2<x<1$. In other words, when the concentration of Ge is within $x 1<x<x 2$, the alloy becomes unstable and decomposes into components $x 1$ and $\mathrm{x} 2$ phases. 
On the other hand, the growth of $\mathrm{Ge}_{\mathrm{x}} \mathrm{Sn}_{1-\mathrm{x}} \mathrm{Se}$ alloys is believed as a cation exchange mechanism [38], which means that nucleation begins as SnSe (or a tin-rich selenide) and gradually incorporates Ge over the growth period. Thermodynamic driving force for the exchange can be evaluated from the calculations of substitution energy, which is defined as

$$
E_{s}=E(\text { doped })-E(\text { pure })-n \mu_{G e}+n \mu_{S n}
$$

where $E$ (doped) and $E$ (pure) are the total energies of the Ge-doped and pure SnSe supercells, $\mu_{\mathrm{Ge}}$ and $\mu_{\mathrm{Sn}}$ represent the chemical potentials of the Ge and Sn atoms, respectively. From this definition, the more negative of the $E_{S}$, the easier of the alloy formation. The calculated substitution energies are listed in Table 1 . These values are all considerably negative, suggesting that the Ge-Sn exchange mechanism accounts for the formation of alloys.

Table 1. Substitution Energies (in eV) of the $\mathrm{Ge}_{x} \mathrm{Sn}_{1-\mathrm{x}} \mathrm{Se}$ Alloys with Different Ge Contents.

\begin{tabular}{ccccc}
\hline $\mathbf{x}$ & $\mathbf{0 . 2 5}$ & $\mathbf{0 . 5 0}$ & $\mathbf{0 . 7 5}$ & $\mathbf{1 . 0 0}$ \\
\hline$E_{s}$ & -3.77 & -7.77 & -11.87 & -16.21 \\
\hline
\end{tabular}

\subsection{Electronic Properties of $\mathrm{Ge}_{x} \mathrm{Sn}_{1-x}$ Se Alloys}

Next, electronic properties of the alloys are investigated with the variation of Ge concentration x. As shown in Figure 3a, the band gaps of SnSe and GeSe are calculated to be 0.92 (indirect) and $1.13 \mathrm{eV}$ (direct), respectively, which are well consistent with the results calculated at the same level, e.g., 0.96 and $1.18 \mathrm{eV}$ [50]. It is observed that as the composition of the $\mathrm{Ge}_{\mathrm{x}} \mathrm{Sn}_{1-\mathrm{x}}$ Se alloy becomes more Ge rich, the band gap gradually increases. Interestingly, this increase is approximately linear from $x=0$ to $x=0.75$, but displays a notable bending at $x=1$. To analyze the reason for this observation, the band edge positions referenced to the vacuum energy levels of the alloys are given in Figure $3 \mathrm{~b}$. It can be seen that the positions of conduction band minimum (CBM) is almost invariable and linearly varied, while those of valence band maximum (VBM) decreases with less linearity, especially at the $x=1$. Examining the partial charge densities of the alloys with the selected Ge concentrations shown in Figure 4, one can see that the densities at the CBM are always delocalized and evenly distributed. However, the densities at the VBM dominantly distribute around the Se atom, with a small distribution on $\mathrm{Sn} / \mathrm{Ge}$ atoms, which seems thinner as the Ge concentration increases.
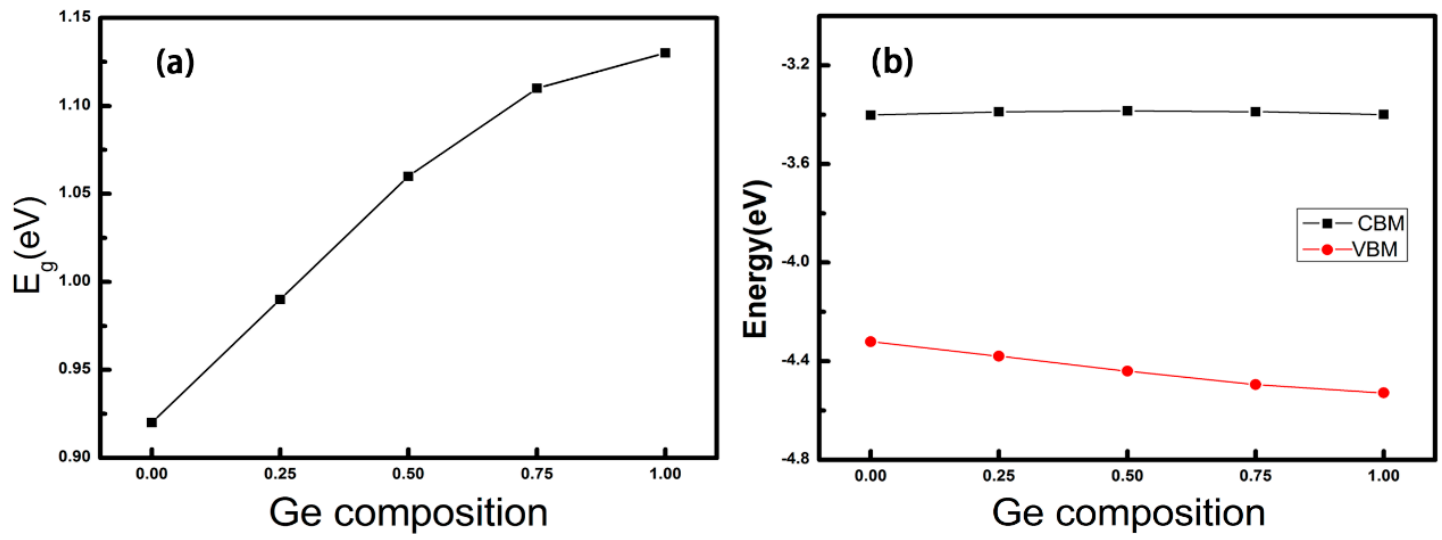

Figure 3. Calculated Band Gaps (a) and Band Edge Positions with Respect to Vacuum Energy Level (b) of $\mathrm{Ge}_{\mathrm{x}} \mathrm{Sn}_{1-\mathrm{x}} \mathrm{Se}$ Monolayers as a Function of the Ge Concentration. 


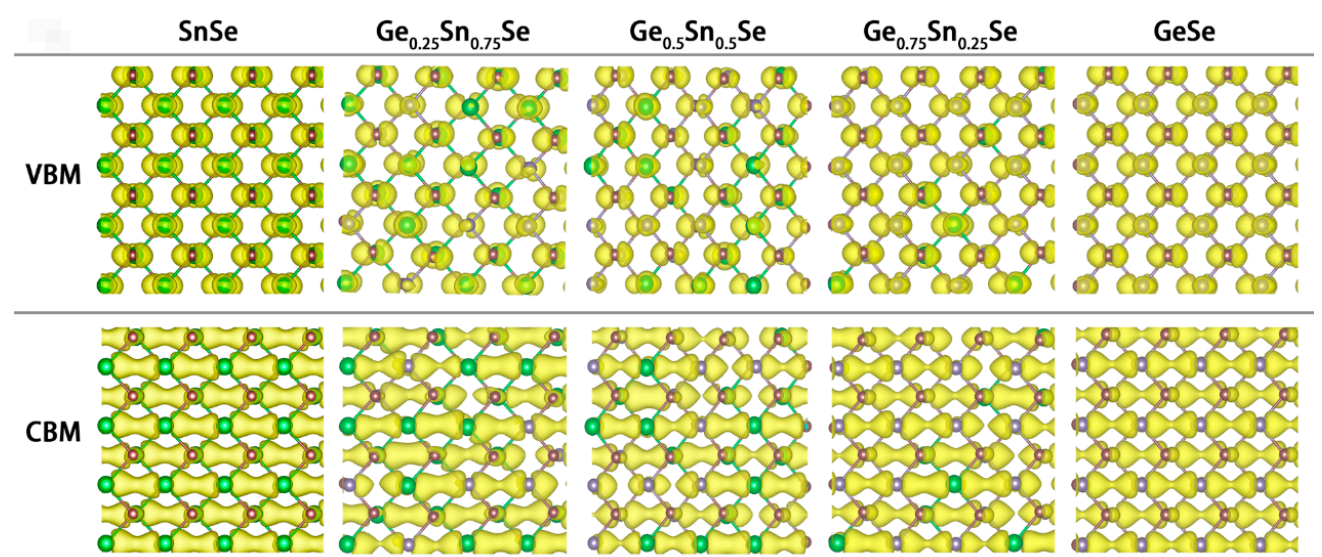

Figure 4. Partial Charge Densities at the VBM and CBM States for the Selected Alloys, where Green, Purple, and Grey Balls Represent Sn, Se and Ge Atoms, respectively.

Moreover, from Figure 3b, the increase of band gap with the Ge concentration is due to the decline of VBM edge position. To get further insight for this variation, the atom-projected band structures (atom contributions are indicated by different colors) and the density of states (DOS) are illustrated in Figure 5. As is seen, the atom contribution to the CBM changes from Sn to Ge, while the VBM is contributed by the hybridization of both $\mathrm{Sn} / \mathrm{Ge}$ and Se atoms. This situation is different from the transition metal alloy systems, in which the VBM states are uniformly distributed among W and Mo d-orbitals [31]. Scrutinizing the DOS, for CBM, the main contribution of SnSe is from Sn-p orbital. With the concentration of Ge reaches 0.75 , the main contribution changes to Ge-p orbital. While CBM states experience a bigger change of atom contribution, the energy distribution is nearly unchanged. This situation is because the formed anti-bonding $\pi^{*}$ orbital may suffer a less energy disturbation with the orbital composition change. In contrast, for the VBM, the contribution seems like from the Se-p orbit all the time, along with the considerable contribution from the hybridization of s- and p-orbitals of $\mathrm{Sn} / \mathrm{Ge}$. As the electronegativity difference between Ge (2.01) and Se (2.55) is smaller than that of between Sn (1.96) and Se, the resulting weaker bonding at the VBM with the Ge concentration increasing gives rise to the energy of VBM uplift.
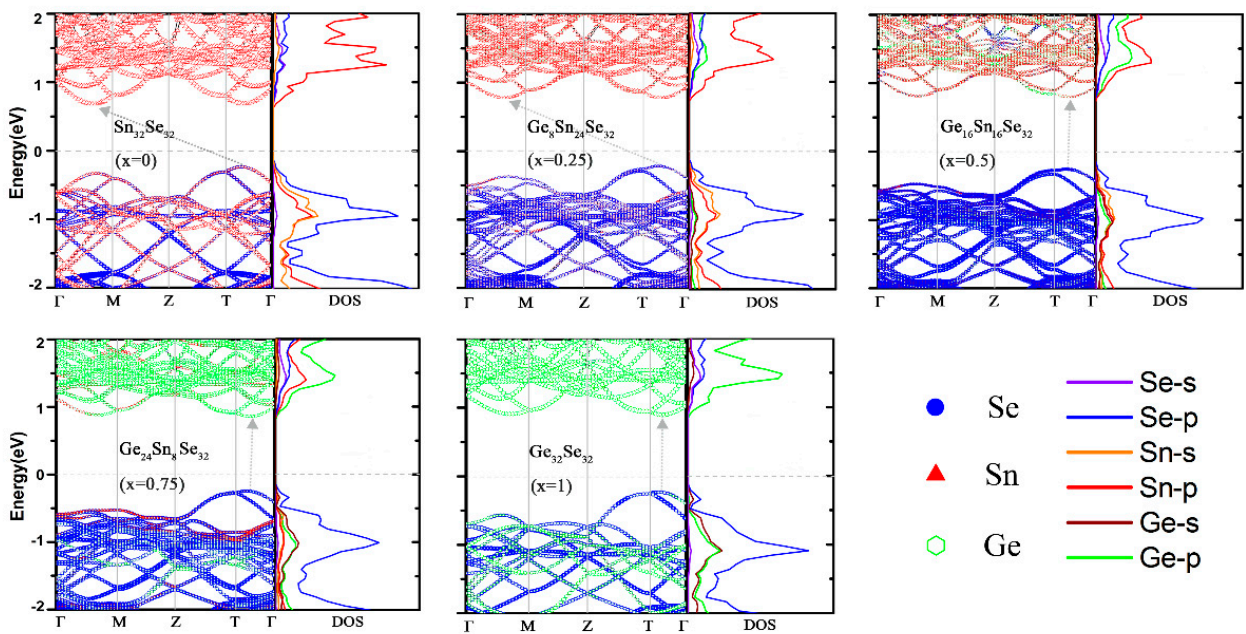

Figure 5. Atom-projected Band Structures and Density of States of the Different $\mathrm{Ge}_{\mathrm{x}} \mathrm{Sn}_{1-\mathrm{x}} \mathrm{Se}(\mathrm{x}=0.0$, $0.25,0.5,0.75$, and 1.0 ) alloys. The direct/indirect bandgap is indicated by the grey arrow.

Another interesting finding is the indirect to direct bandgap crossover occurs at the Ge concentration of 0.5. As is seen, SnSe is found to have an indirect bandgap, but with the Ge 
concentration increasing to 0.5 , the alloy turns into a direct bandgap as the GeSe monolayer. While experimental investigations focusing on mono and few-layer SnSe and GeSe are scarce, the indirect/direct bandgap feature of SnSe/GeSe monolayers has been validated by previous reported theoretical results [50-52]. It is known that 2D materials with the direct bandgap is urgently needed as electrons can be directly excited from the VBM to the CBM by the incident light with an appropriate frequency without the aid of phonons, which leads to the potential application in the optical devices. Moreover, our Heyd-Scuseria-Ernzerhof (HSE06) [53] calculations give the band gaps of 1.39 and $1.59 \mathrm{eV}$ for SnSe and GeSe monolayer, respectively, which are larger than those calculated at the PBE level whereas the band structure topologies are almost the same, with the main difference of CBM state upshift. Note that the HSE06 function used here is only to obtain more accurate band gap as the predicted band gaps of GeSe, SnSe bulks are very close to the experimental ones $[50,52,54]$. For example, the band gaps measured by Kim and Choi are $0.88 \mathrm{eV}$ for SnSe single crystal and $1.10 \mathrm{eV}$ for GeSe [54]. Theoretically, Shi and Kioupakis obtained the band gaps of $0.89 \mathrm{eV}$ and $1.10 \mathrm{eV}$ for SnSe and GeSe bulks at the HSE06 level, respectively [52]. Therefore, through forming alloys with $x \geq 0.5$, on the one hand, the alloy becomes the direct bandgap semiconductor which facilitates the efficiency of light absorption; on the other hand, the alloy has the perfect band gap ranging from 1.50 to $1.59 \mathrm{eV}$, which is the most optimal value for the materials applied in solar cells.

\subsection{Optical Properties of $\mathrm{Ge}_{x} \mathrm{Sn}_{1-x}$ Se Alloys}

As described above, $\mathrm{Ge}_{\mathrm{x}} \mathrm{Sn}_{1-\mathrm{x}} \mathrm{Se}$ alloys have the band gap ranging from 0.92 to $1.13 \mathrm{eV}$ at the PBE level or from 1.39 to $1.59 \mathrm{eV}$ at the HSE06 level. Note that these variation scopes cover the main solar irradiation of the visible range. To further validate the potential applications on the photoelectronics and photovoltaics, optical absorption properties are conducted according to the Kramer-Kroing relationship [55]. The calculated optical absorption coefficients with the incident light polarization along the $y$ and $z$ directions are presented in Figure 6. First, an anisotropy feature is observed as the absorption coefficients along the y direction (armchair), which are significantly larger than those of $z$ direction (zigzag). Second, with the Ge concentration increasing, the optical absorption edges along the $y$ and $z$ directions are blue-shifted, which is consistent with the order of the calculated band gaps. Third, the absorption coefficients reach around $10^{5} \mathrm{~cm}^{-1}$ when the photo energy is larger than $2.0 \mathrm{eV}$, especially the absorption strength is significantly enhanced through alloying. These observations notably verify that through alloying GeSe and SnSe, the optical properties can be improved by subtly controlling the compositions.

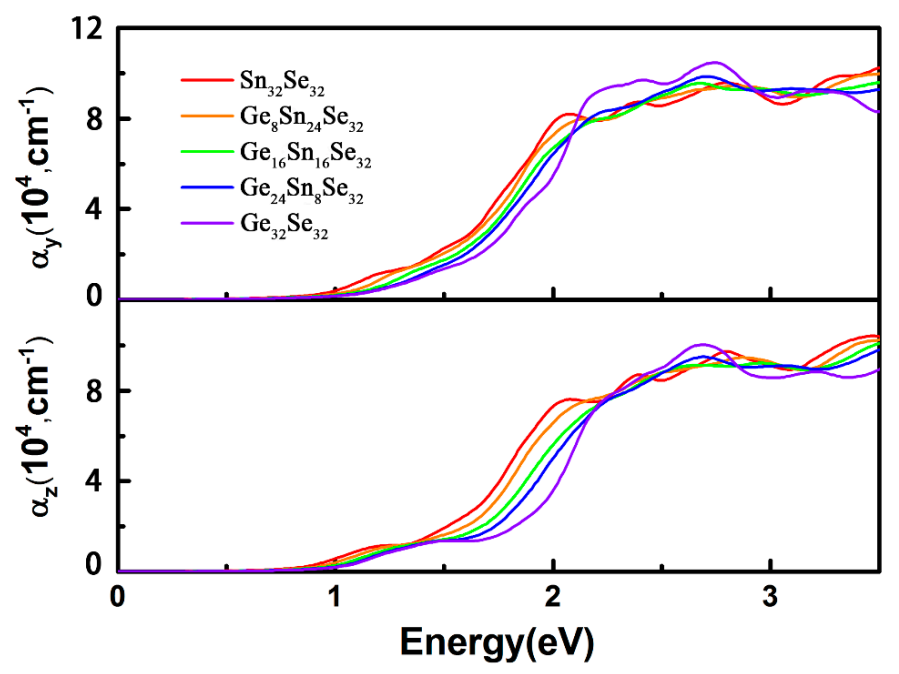

Figure 6. Optical Absorption Coefficients along the $y$ and $z$ Directions of the $\mathrm{Ge}_{\mathrm{x}} \mathrm{Sn}_{1-\mathrm{x}} \mathrm{Se}$ Alloys with Different Ge Compositions. 


\section{Conclusions}

In summary, we have systematically studied the stabilities, electronic, and optical properties of $\mathrm{Ge}_{\mathrm{x}} \mathrm{Sn}_{1-\mathrm{x}} \mathrm{Se}$ alloys with the variation of Ge content through first-principles calculation. The structures of alloys were first generated by the tool of ATAT. Thermodynamic analysis show that the alloys are considerable stable, and the soluble temperature of GeSe and SnSe is estimated to be $580 \mathrm{~K}$. The negative substitution energies of Ge replacing Sn in the SnSe monolayer verify the proposed cation exchange mechanism for the alloy growth. Electronic property calculations show that with the Ge concentration increasing, the band gap of alloys ranges from 1.39 to $1.59 \mathrm{eV}$ at the HSE06 level. The non-linear increase of band gap, especially at the $x=1$, is due to the uneven distribution of charge densities at the VBM states. Interestingly, when Ge concentration exceeds 0.5, on the one hand, the alloys have the direct bandgap which facilitates the efficiency of light absorption; on the other hand, the alloys have perfect band gaps in the range of 1.50-1.59 eV which falls into the most optimal scope for the application in solar cells. Optical calculations further verify that through alloying, the optical properties can be improved by subtly controlling the compositions. We believe that our work may shed light on the future applications of $\mathrm{Ge}_{\mathrm{x}} \mathrm{Sn}_{1-\mathrm{x}} \mathrm{Se}$ alloys in optoelectronics.

Author Contributions: Investigation, Q.Q.; methodology, Q.Q., L.P., Y.C. and L.S.; writing—original draft preparation, Q.Q.; writing—review and editing, Q.Q., J.D. and Y.H.; funding acquisition, J.D., Y.H.

Funding: This research was funded by National Natural Science Foundation of China, grant numbers 21573002 (Y.H.) and 21705003 (J.D.).

Acknowledgments: The numerical calculations in this paper have been done on the super-computing system in the Supercomputing Center of University of Science and Technology of China.

Conflicts of Interest: The authors declare no conflict of interest.

\section{References}

1. Novoselov, K.S.; Geim, A.K.; Morozov, S.V.; Jiang, D.; Zhang, Y.; Dubonos, S.V.; Grigorieva, I.V.; Firsov, A.A. Electric Field Effect in Atomically Thin Carbon Films. Science 2004, 306, 666-669. [CrossRef] [PubMed]

2. Bolotin, K.I.; Sikes, K.J.; Jiang, Z.; Klima, M.; Fudenberg, G.; Hone, J.; Kim, P.; Stormer, H.L. Ultrahigh Electron Mobility in Suspended Graphene. Solid State Commun. 2008, 146, 351-355. [CrossRef]

3. Novoselov, K.S.; Geim, A.K.; Morozov, S.; Jiang, D.; Katsnelson, M.; Grigorieva, I.; Dubonos, S.; Firsov, A.A. Two-Dimensional Gas of Massless Dirac Fermions in Graphene. Nature 2005, 438, 197. [CrossRef] [PubMed]

4. Novoselov, K.S.; Jiang, D.; Schedin, F.; Booth, T.J.; Khotkevich, V.V.; Morozov, S.V.; Geim, A.K. Two-Dimensional Atomic Crystals. Proc. Natl. Acad. Sci. USA 2005, 102, 10451-10453. [CrossRef] [PubMed]

5. Fiori, G.; Bonaccorso, F.; Iannaccone, G.; Palacios, T.; Neumaier, D.; Seabaugh, A.; Banerjee, S.K.; Colombo, L. Electronics Based on Two-Dimensional Materials. Nat. Nanotechnol. 2014, 9, 768-779. [CrossRef] [PubMed]

6. Wang, Q.H.; Kalantarzadeh, K.; Kis, A.; Coleman, J.N.; Strano, M.S. Electronics and Optoelectronics of Two-Dimensional Transition Metal Dichalcogenides. Nat. Nanotechnol. 2012, 7, 699-712. [CrossRef] [PubMed]

7. Qiao, J.; Kong, X.; Hu, Z.X.; Yang, F.; Ji, W. High-Mobility Transport Anisotropy and Linear Dichroism in Few-Layer Black Phosphorus. Nat. Commun. 2014, 5, 4475. [CrossRef] [PubMed]

8. Shi, Y.; Hamsen, C.; Jia, X.; Kim, K.K.; Reina, A.; Hofmann, M.; Hsu, A.L.; Zhang, K.; Li, H.; Juang, Z.Y. Synthesis of Few-Layer Hexagonal Boron Nitride Thin Film by Chemical Vapor Deposition. Nano Lett. 2010, 10, 4134. [CrossRef] [PubMed]

9. Naguib, M.; Mochalin, V.N.; Barsoum, M.W.; Gogotsi, Y. 25th Anniversary Article: Mxenes: A New Family of Two-Dimensional Materials. Adv. Mater. 2013, 26, 992-1005. [CrossRef] [PubMed]

10. Zhang, S.; Yan, Z.; Li, Y.; Chen, Z.; Zeng, H. Atomically Thin Arsenene and Antimonene: SemimetalSemiconductor and Indirect-Direct Band-Gap Transitions. Angew. Chem. 2015, 127, 3155-3158. [CrossRef]

11. Pumera, M.; Sofer, Z. 2d Monoelemental Arsenene, Antimonene, and Bismuthene: Beyond Black Phosphorus. Adv. Mater. 2017, 29, 1605299. [CrossRef] [PubMed]

12. Vogt, P.; De Padova, P.; Quaresima, C.; Avila, J.; Frantzeskakis, E.; Asensio, M.C.; Resta, A.; Ealet, B.; Le Lay, G. Silicene: Compelling Experimental Evidence for Graphenelike Two-Dimensional Silicon. Phys. Rev. Lett. 2012, 108, 155501. [CrossRef] [PubMed] 
13. Ren, C.-C.; Zhang, S.-F.; Ji, W.-X.; Zhang, C.-W.; Li, P.; Wang, P.-J. Tunable Electronic and Topological Properties of Germanene by Functional Group Modification. Nanomaterials 2018, 8, 145. [CrossRef] [PubMed]

14. Zhu, Z.; Cai, X.; Yi, S.; Chen, J.; Dai, Y.; Niu, C.; Guo, Z.; Xie, M.; Liu, F.; Cho, J.-H.; et al. Multivalency-Driven Formation of Te-Based Monolayer Materials: A Combined First-Principles and Experimental Study. Phys. Rev. Lett. 2017, 119, 106101. [CrossRef] [PubMed]

15. Radisavljevic, B.; Radenovic, A.; Brivio, J.; Giacometti, V.; Kis, A. Single-Layer MoS 2 Transistors. Nat. Nanotechnol. 2011, 6, 147-150. [CrossRef] [PubMed]

16. Pan, H. Progress on the Theoretical Study of Two-Dimensional MoS 2 Monolayer and Nanoribbon; Springer International Publishing: Cham, Switzerland, 2014; pp. 992-995.

17. Wei, X.L.; Zhang, H.; Guo, G.C.; Li, X.B.; Lau, W.M.; Liu, L.M. Modulating the Atomic and Electronic Structures through Alloying and Heterostructure of Single-Layer $\mathrm{MoS}_{2}$. J. Mater. Chem. A 2014, 2, 2101-2109. [CrossRef]

18. Mak, K.F.; Lee, C.; Hone, J.; Shan, J.; Heinz, T.F. Atomically Thin $\mathrm{MoS}_{2}$ : A New Direct-Gap Semiconductor. Phys. Rev. Lett. 2010, 105, 136805. [CrossRef] [PubMed]

19. Yang, L.; Majumdar, K.; Liu, H.; Du, Y.; Wu, H.; Hatzistergos, M.; Hung, P.Y.; Tieckelmann, R.; Tsai, W.; Hobbs, C. Chloride Molecular Doping Technique on 2d Materials: $\mathrm{WS}_{2}$ and $\mathrm{MoS}_{2}$. Nano Lett. 2014, 14, 6275. [CrossRef] [PubMed]

20. Low, T.; Rodin, A.S.; Carvalho, A.; Jiang, Y.; Wang, H.; Xia, F.; Castro Neto, A.H. Tunable Optical Properties of Multilayer Black Phosphorus Thin Films. Phys. Rev. B 2014, 90, 075434. [CrossRef]

21. Xia, F.; Wang, H.; Jia, Y. Rediscovering Black Phosphorus as an Anisotropic Layered Material for Optoelectronics and Electronics. Nat. Commun. 2014, 5, 4458. [CrossRef] [PubMed]

22. Sun, Y.; Fujisawa, K.; Lin, Z.; Lei, Y.; Mondschein, J.S.; Terrones, M.; Schaak, R.E. Low-Temperature Solution Synthesis of Transition Metal Dichalcogenide Alloys with Tunable Optical Properties. J. Am. Chem. Soc. 2017, 139, 11096-11105. [CrossRef] [PubMed]

23. Wang, J.; Yang, T.; Zhang, Z.; Yang, L. Enhanced Doping Effect on Tuning Structural Phases of Monolayer Antimony. Appl. Phys. Lett. 2018, 112, 213104. [CrossRef]

24. Wang, Z.; Shen, Y.; Ito, Y.; Zhang, Y.; Du, J.; Fujita, T.; Hirata, A.; Tang, Z.; Chen, M. Synthesizing 1T-1H Two-Phase $\mathrm{Mo}_{1-\mathrm{x}} \mathrm{W}_{\mathrm{x}} \mathrm{S}_{2}$ Monolayers by Chemical Vapor Deposition. ACS Nano 2018, 12, 1571-1579. [CrossRef] [PubMed]

25. Darwish, A.A.A.; Rashad, M.; Bekheet, A.E.; El-Nahass, M.M. Linear and Nonlinear Optical Properties of $\mathrm{GeSe}_{2-\mathrm{x}} \mathrm{Sn}_{\mathrm{x}}(0 \leq \mathrm{x} \leq 0.8)$ Thin Films for Optoelectronic Applications. J. Alloys Compd. 2017, 709, 640-645. [CrossRef]

26. Li, X.; Dai, Y.; Ma, Y.; Han, S.; Huang, B. Graphene/G- $\mathrm{C}_{3} \mathrm{~N}_{4}$ Bilayer: Considerable Band Gap Opening and Effective Band Structure Engineering. Phys. Chem. Chem. Phys. 2014, 16, 4230-4235. [CrossRef] [PubMed]

27. Huo, N.; Kang, J.; Wei, Z.; Li, S.-S.; Li, J.; Wei, S.-H. Novel and Enhanced Optoelectronic Performances of Multilayer $\mathrm{MoS}_{2}-\mathrm{WS}_{2}$ Heterostructure Transistors. Adv. Funct. Mater. 2014, 24, 7025-7031. [CrossRef]

28. Sapna, S.; Yuya, T.; Hisanori, S.; Ryo, K. Simple Fabrication of Air-Stable Black Phosphorus Heterostructures with Large-Area Hbn Sheets Grown by Chemical Vapor Deposition Method. 2D Materials 2016, 3, 035010.

29. Huang, Y.; Chen, X.; Zhou, D.; Liu, H.; Wang, C.; Du, J.; Ning, L.; Wang, S. Stabilities, Electronic and Optical Properties of $\mathrm{SnSe}_{2(1-\mathrm{x})} \mathrm{S}_{2 \mathrm{x}}$ Alloys: A First-Principles Study. J. Phys. Chem. C 2016, 120, 5839-5847. [CrossRef]

30. Wang, Y.; Le Huang, L.H.; Li, B.; Shang, J.; Xia, C.; Fan, C.; Deng, H.-X.; Wei, Z.; Li, J. Composition-Tunable $2 \mathrm{~d} \mathrm{SnSe}{ }_{2(1-x)} \mathrm{S}_{2 \mathrm{x}}$ Alloys Towards Efficient Bandgap Engineering and High Performance (Opto)Electronics. J. Mater. Chem. C 2017, 5, 84-90. [CrossRef]

31. Tongay, S.; Narang, D.S.; Kang, J.; Fan, W. Two-Dimensional Semiconductor Alloys: Monolayer $\mathrm{Mo}_{1-\mathrm{x}} \mathrm{W}_{\mathrm{x}} \mathrm{Se}_{2}$. Appl. Phys. Lett. 2014, 104, 012101. [CrossRef]

32. Zheng, S.; Sun, L.; Yin, T.; Dubrovkin, A.M.; Liu, F.; Liu, Z.; Shen, Z.X.; Fan, H.J. Monolayers of $\mathrm{W}_{\mathrm{x}} \mathrm{Mo}_{1-\mathrm{x}} \mathrm{S}_{2}$ Alloy Heterostructure with in-Plane Composition Variations. Appl. Phys. Lett. 2015, 106, 063113. [CrossRef]

33. Tan, W.; Wei, Z.; Liu, X.; Liu, J.; Fang, X.; Fang, D.; Wang, X.; Wang, D.; Tang, J.; Fan, X. Ordered and Disordered Phases in $\mathrm{Mo}_{1-\mathrm{x}} \mathrm{W}_{\mathrm{x}} \mathrm{S}_{2}$ Monolayer. Sci. Rep. 2017, 7, 15124. [CrossRef] [PubMed]

34. Guo, S.; Yuan, L.; Liu, X.; Zhou, W.; Song, X.; Zhang, S. First-Principles Study of $\mathrm{SO}_{2}$ Sensors Based on Phosphorene and Its Isoelectronic Counterparts: GeS, GeSe, SnS, SnSe. Chem. Phys. Lett. 2017, 686, 83-87. [CrossRef] 
35. Peng, L.; Wang, C.; Qian, Q.; Bi, C.; Wang, S.; Huang, Y. Complete Separation of Carriers in the GeS/SnS Lateral Heterostructure by Uniaxial Tensile Strain. ACS. Appl. Mater. Interfaces 2017, 9, 40969-40977. [CrossRef] [PubMed]

36. Shafique, A.; Shin, Y.H. Thermoelectric and Phonon Transport Properties of Two-Dimensional IV-VI Compounds. Sci. Rep. 2017, 7, 506. [CrossRef] [PubMed]

37. Deb, A.K.; Kumar, V. Bandgap Engineering in Semiconducting One to Few Layers of SnS and SnSe. Phys. Status Solidi B Basic Res. 2017, 254, 1600379. [CrossRef]

38. Buckley, J.J.; Rabuffetti, F.A.; Hinton, H.L.; Brutchey, R.L. Synthesis and Characterization of Ternary $\mathrm{Sn}_{\mathrm{x}} \mathrm{Ge}_{1-\mathrm{x}} \mathrm{Se}$ Nanocrystals. Chem. Mater. 2012, 24, 3514-3516. [CrossRef]

39. Fu, Y.; Xu, J.; Liu, G.-Q.; Tan, X.; Liu, Z.; Wang, X.; Shao, H.; Jiang, H.; Liang, B.; Jiang, J. Study on Thermoelectric Properties of Polycrystalline SnSe by Ge Doping. J. Electron. Mater. 2017, 46, 3182-3186. [CrossRef]

40. Kresse, G.; Hafner, J. Ab Initio Molecular Dynamics for Liquid Metals. Phys Rev. B Condens. Matter. 1993, 48, 558-561. [CrossRef]

41. Kresse, G.; Furthmüller, J. Efficiency of Ab-Initio Total Energy Calculations for Metals and Semiconductors Using a Plane-Wave Basis Set. Comp. Mater. Sci. 1996, 6, 15-50. [CrossRef]

42. PE, B. Projector Augmented-Wave Method. Phys. Rev. B Condens. Matter. 1994, 50, 17953-17979.

43. Perdew, J.P.; Wang, Y. Accurate and Simple Analytic Representation of the Electron-Gas Correlation Energy. Phys. Rev. B Condens. Matter. 1992, 45, 13244. [CrossRef] [PubMed]

44. Perdew, J.P.; Chevary, J.A.; Vosko, S.H.; Jackson, K.A.; Pederson, M.R.; Singh, D.J.; Fiolhais, C. Atoms, Molecules, Solids, and Surfaces: Applications of the Generalized Gradient Approximation for Exchange and Correlation. Phys. Rev. B Condens. Matter. 1993, 46, 6671-6687. [CrossRef]

45. Monkhorst, H.J. Special Points for Brillouin-Zone Integrations. Phys. Rev. B Condens. Matter. 1976, 16, 1748-1749. [CrossRef]

46. Zunger, A.; Wei, S.H.; Ferreira, L.G.; Bernard, J.E. Special Quasirandom Structures. Phys. Rev. Lett. 1990, 65, 353-356. [CrossRef] [PubMed]

47. Van de Walle, A.; Asta, M.; Ceder, G. The Alloy Theoretic Automated Toolkit: A User Guide. Calphad 2002, 26, 539-553. [CrossRef]

48. Denton, A.R.; Ashcroft, N.W. Vegard's Law. Phys. Rev. A 1991, 43, 3161. [CrossRef] [PubMed]

49. Andrews, K.W. Alloy Phase Equilibria; Elsevier Pub. Co.: Amsterdam, The Netherlands, 1966; pp. $255-256$.

50. Gomes, L.C.; Carvalho, A. Phosphorene Analogues: Isoelectronic Two-Dimensional Group-IV Monochalcogenides with Orthorhombic Structure. Phys. Rev. B 2015, 92, 085406. [CrossRef]

51. Fei, R.; Li, W.; Li, J.; Yang, L. Giant Piezoelectricity of Monolayer Group IV Monochalcogenides: SnSe, SnS, GeSe, and GeS. Appl. Phys. Lett. 2015, 107, 173104. [CrossRef]

52. Shi, G.; Kioupakis, E. Anisotropic Spin Transport and Strong Visible-Light Absorbance in Few-Layer SnSe and GeSe. Nano Lett. 2015, 15, 6926-6931. [CrossRef] [PubMed]

53. Heyd, J.; Scuseria, G.E.; Ernzerhof, M. Hybrid Functionals Based on a Screened Coulomb Potential. J. Chem. Phys. 2003, 118, 8207-8215. [CrossRef]

54. Kim, Y.; Choi, I.-H. Optical and Electrical Properties of GeSe and SnSe Single Crystals. J. Korean Phys. Soc. 2018, 72, 238-242. [CrossRef]

55. Ehrenreich, H.; Cohen, M.H. Self-Consistent Field Approach to the Many-Electron Problem. Phy. Rev. 1959, 115, 786-790. [CrossRef]

(C) 2018 by the authors. Licensee MDPI, Basel, Switzerland. This article is an open access article distributed under the terms and conditions of the Creative Commons Attribution (CC BY) license (http:/ / creativecommons.org/licenses/by/4.0/). 